\title{
Analysis transmission performance of complex interconnect structure and equivalent circuit
}

\author{
Yuling Shang ${ }^{1}$, Jianfeng $\mathrm{Ma}^{1}$, Chunquan $\mathrm{Li}^{2, *}$ \\ ${ }^{1}$ Department of Electronic Engineering and Automation, Guilin University of Electronic Technology, \\ Guilin, 541004, China \\ ${ }^{2}$ Department of Electromechanical engineering, Guilin University of Electronic Technology, Guilin, \\ 541004, China
}

Keywords: complex interconnect structure model; transmission characteristic; circuit model; Sparameter.

\begin{abstract}
The signal integrity problem has become a limit on current electronic system design and development. This paper studies the transmission performance for high-speed PCB complex interconnect structural model consists of via, solder ball and trace. Different length of traces and different radius of pads were simulated using HFSS to evaluate the effect of these parameters on the transmission characteristic. An equivalent circuit model of the complex interconnect structure was presented and the parameters values were extracted. The circuit model was simulated using the software ADS, compared to the results simulated using HFSS, the differences of return loss S11were within $1 \mathrm{~dB}$ ranging from $1 \mathrm{GHz}$ to $6 \mathrm{GHz}$, and within $2 \mathrm{~dB}$ ranging from $6 \mathrm{GHz}$ to $10 \mathrm{GHz}$.
\end{abstract}

\section{Introduction}

Today's integrated circuits develop toward high performance, high density and highly integrated, but Signal integrity ${ }^{[1]}$ issues have become a problem which cannot be ignored. The complex interconnect structure consists of via ${ }^{[2]}$, solder ball ${ }^{[3]}$ and trace has become the basic unit of high density integrated circuits. Effect of the via and solder ball on the signal transmission can be negligible at low operating frequency, but when the operating frequency rises up to the RF or microwave segment, the complex interconnect structure is an entire signal transmission path characteristics of the transmission line exhibition. Via provide a connection between the signal layers, the BGA (Ball Grid Array, abbr. BGA) solder ball is the connection between the BGA package device and the circuit board. Via and solder ball are discontinuous factors on the transmission path, which will seriously affect the quality of the signal propagation. Therefore, it is necessary at high frequencies the research on the signal integrity issues caused by the complex interconnect structure consisting of via, solder ball and trace as the whole object.

\section{Signal integrity}

Signal integrity is broadly describe the quality of signal transmission in high-speed circuit, which major research the effect of electrical parameters characteristics on signal transmission. Signal propagation always affected the transient impedance on transmission path, when the impedance unchanged the signal is not reflected, otherwise, the signal reflection occurs at the place where the impedance changed. The reflection large enough will cause circuit malfunction.

To solve signal integrity issues of high-speed circuit, the signal integrity simulation must be performed. With reflection questions for via and solder ball continuous research and improvement, foreign scholars have proposed a variety of new via structure, from a single via model to the many vias transition model ${ }^{[4]}$, even Though Silicon Via fault model ${ }^{[5]}$. Research on the BGA solder ball from a single analysis to the grounding solder ball shielding ${ }^{[6]}$, and even differential solder balls performance ${ }^{[7]}$. Domestic interconnect structure for electromagnetic analysis is still in the research methods of follow up abroad, more concerned about the manufacturing processes and materials but the research about the effect of the discontinuous region size on reflection is relatively little. Based 
on this background, this paper select reflections caused by the change of discontinuous impedance to analyse a signal transmission performance of the complex interconnect structure model.

\section{Complex interconnect structure model}

Complex interconnect structure model is shown in Fig. 1. Substrate material selects FR4, the dielectric constant of 4.2 , the via radius is $12 \mathrm{mil}$, via pad radius is $16 \mathrm{mil}$, solder ball radius is $15 \mathrm{mil}$, solder ball pad radius is $16 \mathrm{mil}$, scanning frequency range selection 1-10GHz.

In complex interconnect structure constituted by via, solder ball and trace, there are via-trace, and solder ball-trace discontinuous interconnected cells, shown in Fig.2 and Fig.3, in whose contact points have signal transmission discontinuity. Where $\mathrm{c}=\mathrm{a}+\mathrm{b}$, $\mathrm{a}$ is the discrete region pad width, $\mathrm{b}$ is the length of the discontinuous region trace, $\mathrm{c}$ is the length of the discontinuous region.

For complex interconnect structure model, without changing other simulation parameters and conditions and in the same scanning frequency, selecting discontinuous area trace length as a single variable, signal integrity simulation is performed. When the length of the trace connected with via and solder ball is 6mil, 8mil, 10mil, the simulation results are shown in Fig.4 and Fig.5, respectively.

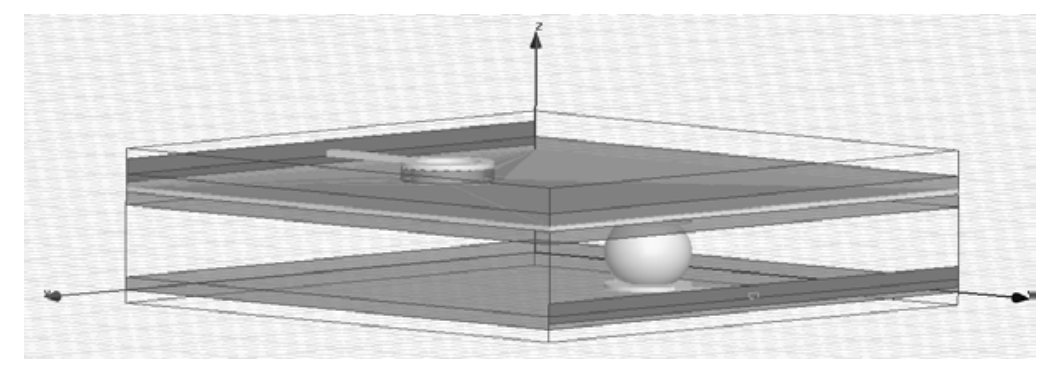

Fig.1: Complex interconnect structure model constituted by via, solder ball and trace

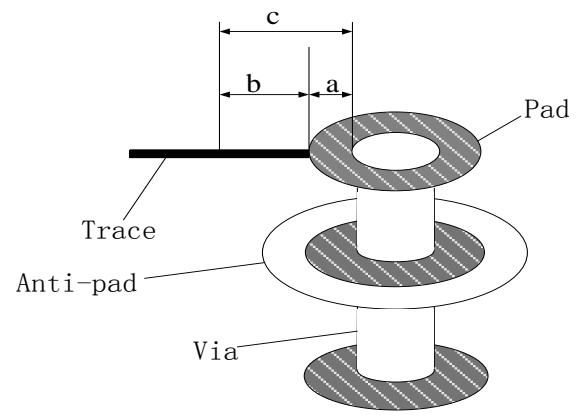

Fig.2: Via - trace discontinuities schematic

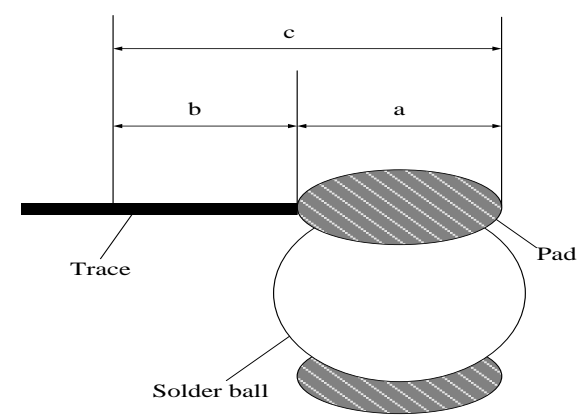

Fig.3: Solder ball - trace discontinuities schematic

As can be seen from the simulation results, with the length of the trace increasing, the return loss $\mathrm{S}_{11}$ of the complex interconnect structure model increases that the signal reflection performance is increasingly strong.

At high frequencies, the current influenced by the skin depth, the resistance is mainly concerned with the frequency. Under the restricting of skin effect, the skin depth is much smaller than the 
geometry thickness of the cross-sectional. Along with frequency increasing, the cross-sectional area of trace where current flows will be decreasing proportional to the square root of the frequency, so that the per unit length impedance of trace increase with frequency proportional to the square root. The general formula $L=0.083 R_{\text {Len }} \sqrt{\varepsilon_{r}}$ of the per unit length inductance of the transmission line indicates, the skin effect cause an increase in resistance that with the trace length $\mathrm{b}$ increasing in the discontinuous region, the transmission line inductance will increase and the return loss $\mathrm{S}_{11}$ becomes larger. As can be seen, the shorter transmission line not only enables signal transmission performance better, but also in line with technological trends.

For complex interconnect structure model, without changing other simulation parameters and conditions and in the same scanning frequency, selecting a pad radius within discontinuous areas as a single variable, signal integrity simulation is performed. When the via pad radius and solder ball pad radius is 16mil, 17mil, 18mil, the simulation results are shown in Fig.6 and Fig.7, respectively.

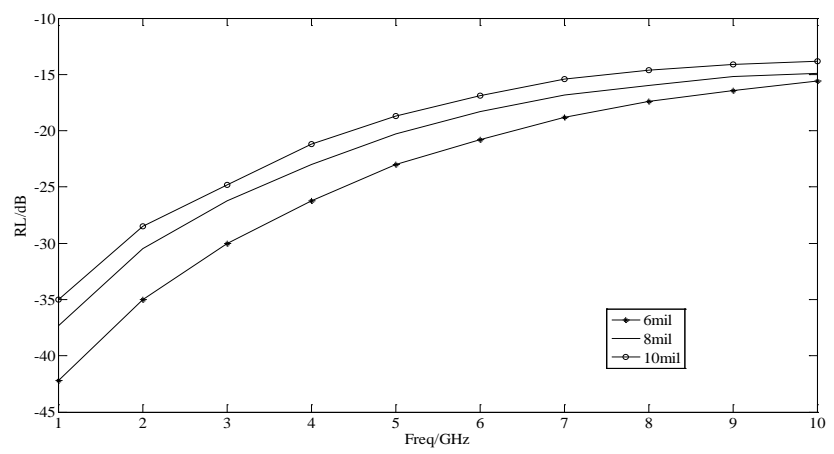

Fig.4: Trace connected with via

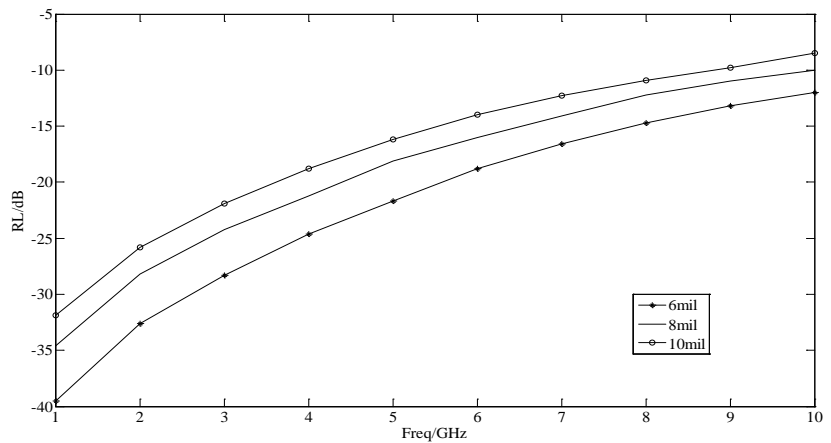

Fig.5: Trace connected with solder ball

As seen from the simulation results, in discontinuous region with the pad radius increases, the return loss $S_{11}$ of the complex interconnect structure model increases, that is to say the signal reflection performance become more and more strong.

For via, the parasitic capacitance to ground is present. By the via parasitic capacitance formula $C=1.41 \varepsilon_{r} T D_{2} /\left(D_{3}-D_{2}\right)$, it is indicated that via parasitic capacitance increases with via pad radius increase, and the resistance caused by the skin effect will increase. By the inductance general formula $L=C \times R^{2}$, the return loss S11 caused by the increase of the via inductance becomes larger.

For solder ball, at high frequencies, the current is mainly distributed in the solder ball surface due to the skin effect. As the frequency increases, the skin depth decreases. From the spherical capacitor formula $C=2 \pi \varepsilon_{0} \varepsilon_{r} / \ln \left(D_{1} /\left(D_{1}-\delta\right)\right)$, with spherical capacitance increasing, the resistance caused by the skin effect will increase, so from the inductance general formula $L=C \times R^{2}$, the influence on the return loss $S_{11}$ caused by the increase of the solder ball inductance becomes larger. 


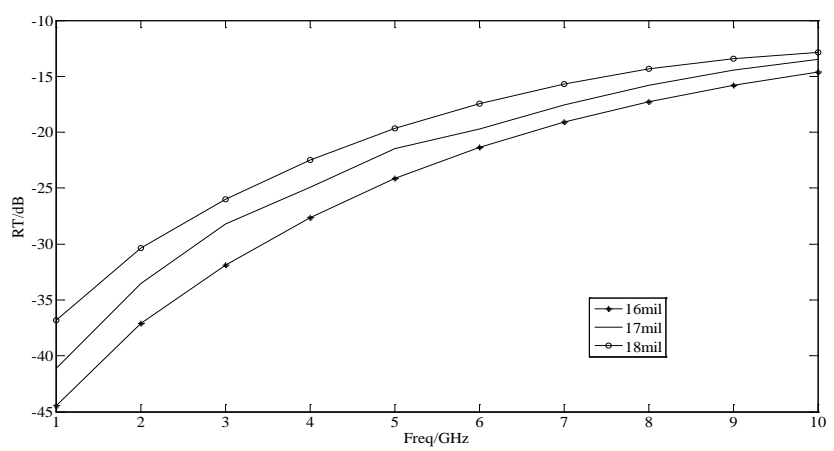

Fig.6: Via pad radius

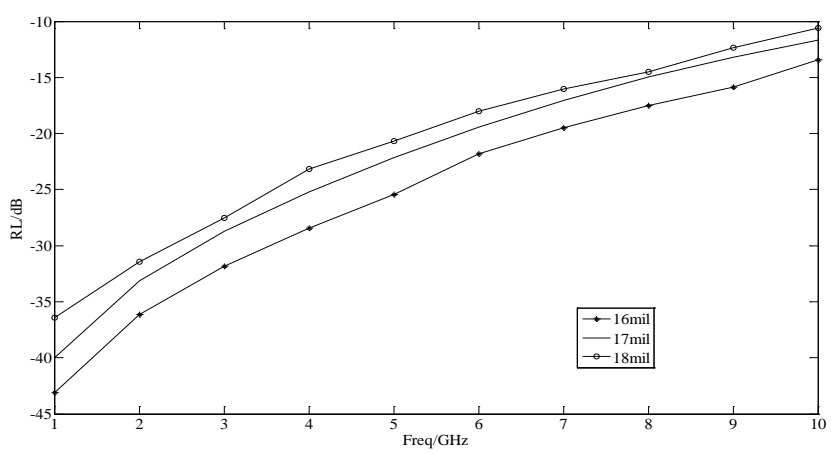

Fig.7: Solder ball pad radius

\section{Equivalent circuit model}

As can be seen from the above simulation results, the impact of changes in the size of the discontinuous region on the signal transmission performance is very important. The equivalent circuit model in this paper is shown in Figure 8.

At high frequencies, the current distribution of the entire signal transmission path should be subject to the skin effect, the current on the trace is mainly distributed over the surface in contact with the air, the via current is mainly distributed on the wall in contact with the air, the solder ball current is mainly distributed on the outer spherical surface in contact with air, the thickness of the current arrangement is mainly skin depth $\delta$. Skin effect determines the high-frequency resistance and can also cause parasitic effects, resulting changes of the capacitance and inductance parameters. The existence of the via parasitic capacitance and the via parasitic inductance cannot be ignored. For solder ball, spherical parasitic capacitance and parasitic inductance must be considered.

Skin depth:

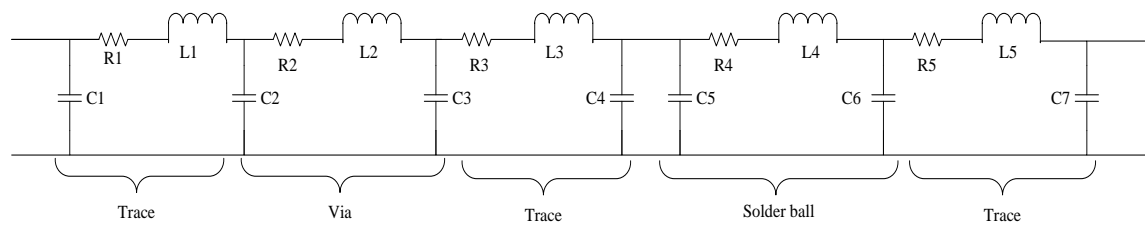

Fig.8: Equivalent circuit model

$$
\delta=\sqrt{1 /\left(\sigma \pi \mu_{0} \mu_{r} t\right)}
$$

Per unit length resistance caused by skin depth:

$$
R_{\text {Len } \delta}=\rho /(w \delta)
$$

Microstrip line unit length capacitance due to skin depth:

$$
\mathrm{C}_{\text {trace }}=\frac{0.67\left(1.41+\varepsilon_{\mathrm{r}}\right)}{\ln (5.98 \mathrm{~h} / 0.8 \mathrm{w}+\mathrm{t})}
$$


Microstrip line unit length inductance due to the skin depth:

$$
L_{\text {traces }}=0.083 R_{\text {Leno } \delta} \sqrt{\varepsilon_{r}}
$$

Via parasitic capacitance:

Via parasitic inductance:

$$
\mathrm{C}_{\text {vial }}=1.41 \varepsilon_{\mathrm{r}} T D_{2} /\left(D_{3}-D_{2}\right)
$$

$$
\mathrm{L}_{\text {vial }}=5.08 \mathrm{~T}\left[\ln \left(4 T / D_{1}\right)\right]
$$

Solder ball parasitic capacitance:

$$
C=2 \pi \varepsilon_{0} \varepsilon_{r} / \ln (r /(r-\delta))
$$

Solder ball parasitic inductance:

$$
\mathrm{L}_{\text {ball1 }}=5 \mathrm{D}[\ln (2 D / r)-3 / 4]
$$

In formulas, $\sigma$ is the electrical conductivity of metal, $\mathrm{u}_{\mathrm{r}}$ is the relative permeability of the wire, $\mathrm{u}_{0}$ is permeability of free space, $\rho$ is the conductivity of the conductor material, $\varepsilon_{r}$ is dielectric constant, $h$ is the thickness of the dielectric layer, $w$ is the trace width, $t$ is the thickness of the trace, $T$ is the height of via, $D_{3}$ is via anti-pad diameter, $D_{2}$ is via pad diameter, $D_{1}$ is via inside diameter, $D$ is the solder ball height, $r$ is the radius of the solder ball. Using Equation (1) - (8) can calculate the parameter values of the elements of the equivalent circuit, $\mathrm{R} 1=\mathrm{R} 3=\mathrm{R} 5=0.076 \mathrm{Ohm}, \mathrm{R} 2=\mathrm{R} 4=0.2 \mathrm{Ohm}$, $\mathrm{L} 1=\mathrm{L} 3=\mathrm{L} 5=0.28 \mathrm{nH}, \mathrm{L} 2=0.85 \mathrm{nH}, \mathrm{L} 4=0.47 \mathrm{nH}, \mathrm{C} 1=\mathrm{C} 4=\mathrm{C} 7=0.068 \mathrm{pF}, \mathrm{C} 2=\mathrm{C} 3=0.3 \mathrm{pF}, \mathrm{C} 5=\mathrm{C} 6=0.2 \mathrm{pF}$.

Using ADS software to get the S-parameters of equivalent circuit model, using HFSS software to get the S-parameters of complex interconnection structure model, compared two methods simulated return loss S11 do not differ in the frequency range $1-6 \mathrm{GHz}$ more than $1 \mathrm{~dB}$, in the $6-10 \mathrm{GHz}$ frequency range differ by no more than $2 \mathrm{~dB}$, as shown in Fig.9.

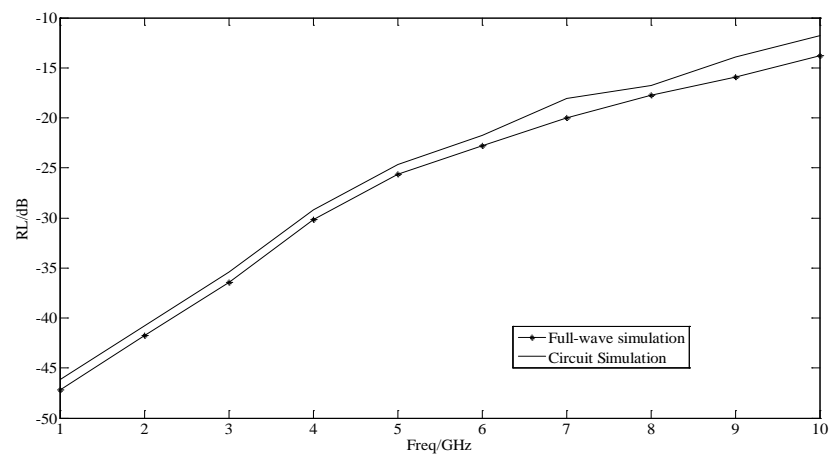

Fig.9: Results comparative validation

\section{Conclusion}

The complex interconnect structure model constituting via, solder ball and trace has become the basic unit of a high-density integrated circuits. As a signal transmission path, signal integrity under high-frequency conditions has become a problem which cannot be ignored. As can be seen from the results in this paper, as the frequency increases, the skin effect is quite large, in discontinuous area trace length and pad width have different degrees of impact on the signal transmission. In discrete areas the smaller size of trace and pad make signal transmission performance better, which is consistent with current trends in technology development. In this paper, an equivalent circuit model is established for the complex interconnect structure model constituting by via, solder ball and trace. The equivalent circuit were verified by the ADS software, the results show that the return loss at 1$6 \mathrm{GHz}$ frequency range S11 within a difference of no more than $1 \mathrm{~dB}$, the difference in the frequency range 6-10GHz not exceed $2 \mathrm{~dB}$. 


\section{Acknowledgments}

The research work reported in this paper is supported by National Natural Science Foundation of China No. 61102012, No.51465013 and No.51165004. Natural Science Foundation of Guangxi Province No. 2012GXNSFBA053176 and No. 2012GXNSFDA053029. Innovation Project of GUET Graduate Education No. GDYCSZ201480.

\section{References}

[1] Shang Yu-Ling. Study on test of crosstalk-induced fault for high speed interconnects Xian: Xidian University, 2009.

[2] Shang Yu-Ling, Qu Li. Analysis for Modeling Electromagnetic Characteristics of Via in HighSpeed Printed Circuit Board. Advance in Information Sciences and Service Sciences, 5(7), pp.589596, 2013.

[3] Shang Yu-Ling, Shi Guang-Yao \& Qu Li. Influence of shape and distribution of BGA solder joints on signal integrity. Journal of Guilin University of Electronic Technology, (33) 4, pp.279-283, 2013.

[4] Casares Miranda F.P., Viereck C. \& Camacho Penalosa C. Vertical microstrip transition for multilayer microwave circuits with decoupled passive and active layers. IEEE Microwave and Wireless Componnents Letters, (16) 7, pp.401-403, 2006.

[5] Kannan S., Kim B. \& Ahn B. Fault Modeling and Multi-Tone Dither Scheme for Testing 3D TSV Defects. Journal of Electronic Testing: Theory and Applications, 28 (1), pp.39-51, 2012.

[6] Kangasvieri T., Halme J. \& Vahakangas J. Broadband BGA-Via Transitions for Reliable RF/Microwave LTCC-SiP Module Packging. IEEE Microwave and Wireless Componnents Letters, 18 (1), pp.34-36, 2008.

[7] Marechal L., Auchere D. \& Imbs Y. BGA package technology considerations for High Speed and RF applications. European Microelectronics Packaging Conference, pp.1-6, 2013. 